\title{
Giant Spin Relaxation Anisotropy in Zinc-Blende Heterostructures
}

\author{
N.S. Averkiev and L.E. Golub \\ A.F. Ioffe Physico-Technical Institute, Russian Academy of Sciences, 194021 St. Petersburg, Russia
}

\begin{abstract}
Spin relaxation in-plane anisotropy is predicted for heterostructures based on zinc-blende semiconductors. It is shown that it manifests itself especially brightly if the two spin relaxation mechanisms (D'yakonov-Perel' and Rashba) are comparable in efficiency. It is demonstrated that for the quantum well grown along the [001] direction, the main axes of spin relaxation rate tensor are [110] and [110].
\end{abstract}

\section{INTRODUCTION}

Spin relaxation processes have significant effect in optical and kinetic properties of semiconductors. They play important role in optical orientation of electrons and nuclei [1] and in anomalous magnetoresistance caused by weak localisation 2]. Both theoretical calculations and experimental data analysis have been carried out assuming that one spin relaxation mechanism dominates only. Therewith in spite of the strong anisotropy of spin-orbit scattering, the relaxation times of spin lying in the plane of a heterostructure with zinc-blende lattice turn out to be independent on orientation with respect to crystallographic axes.

Real heterostructures differ from investigated ideal objects in that several spin relaxation mechanisms exist [3,4]. A spin relaxation mechanism due to only cubic in wave vector terms of the bulk Hamiltonian was investigated for rectangular quantum wells (QWs) in Ref. [5]. It was noted that even in an asymmetrical GaAs QW, the efficiency of another mechanism due to linear in two dimensional wave vector terms is negligibly small. The authors of Ref. [- [- analysing experimental data on anomalous magnetoresistance in InGaAs QWs demonstrated that the both mechanisms may be comparable in efficiency. But in Refs. 3, 4, it was mentioned that the both mechanisms are additive in spin relaxation.

This communication is devoted to an investigation of spin relaxation processes when several mechanisms of spin-orbit scattering exist. We show that contributions of these mechanisms interfere and their simultaneous action leads to the strong anisotropy of spin relaxation even in the plane of a QW.

\section{THEORY}

In zinc-blende semiconductors, spin relaxation of electrons is well known to be due to spin-orbit splitting of conduction band. In a bulk crystal, the splitting is cubic in wave vector. In a QW structure, the corresponding Hamiltonian has to be averaged over the motion along the growth axis. We consider the QW grown along $z$ direction parallel to [001] and choose $x$ and $y$ directions coinciding with crystallographic axes. At relatively small carrier concentrations, one can neglect cubic in 2D wave vector terms and the Hamiltonian has the form:

$$
H_{1}=a_{1}\left(\sigma_{x} k_{x}-\sigma_{y} k_{y}\right) .
$$

Here $\sigma_{i}(i=x, y)$ is the Pauli matrix, $k_{i}$ is the wave vector component in the plane of the $\mathrm{QW}$ and $a_{1}$ is a constant.

In asymmetrical heterostructures, there is a contribution to the Hamiltonian which is absent in the bulk [6]:

$$
H_{2}=a_{2}\left(\sigma_{x} k_{y}-\sigma_{y} k_{x}\right)
$$

where $a_{2}$ is the constant determined by heterointerface properties.

To calculate spin relaxation times, one has to consider the dynamics of spin density matrix, $\rho$ :

$$
\frac{\partial \rho}{\partial t}=-\frac{i}{\hbar}[H, \rho],
$$

where the total two dimensional Hamiltonian is:

$$
H=\frac{\hbar^{2} k^{2}}{2 m}+V+H^{\prime} .
$$

Here $m$ is an effective electron mass, $V(x, y)$ is a scattering potential and

$$
H^{\prime}=H_{1}+H_{2} \text {. }
$$

We assume that the scattering is elastic and independent on spin indices.

Since $H^{\prime}$ is a small perturbation, the spin relaxation times turn out to be much longer than isotropisation times of momentum distribution of electrons. For this reason it is convenient to represent the density matrix as a sum $[7]$ :

$$
\rho=\bar{\rho}+\rho^{\prime}, \quad \overline{\rho^{\prime}}=0,
$$

where the bar means averaging over the directions of $\boldsymbol{k}$. Here $\bar{\rho}$ depends on $\varepsilon=\hbar^{2} k^{2} / 2 m$ and the anisotropic part of the density matrix is due to $H^{\prime}$ only. Hence $\rho^{\prime}$ is proportional to $H^{\prime}$, i.e. $\rho^{\prime}$ is small in comparison to $\bar{\rho}$.

Then in the first order in $H^{\prime}$, the Eq. (3) has the form:

$$
\begin{gathered}
\frac{\partial \rho^{\prime}}{\partial t}=-\frac{i}{\hbar}\left[H^{\prime}, \bar{\rho}\right]-\sum_{\mathbf{k}^{\prime}} W_{\mathbf{k k}^{\prime}}\left[\rho^{\prime}(\boldsymbol{k})-\rho^{\prime}\left(\boldsymbol{k}^{\prime}\right)\right] \\
\frac{\partial \bar{\rho}}{\partial t}=-\frac{i}{\hbar} \overline{\left[H^{\prime}, \rho^{\prime}\right]} .
\end{gathered}
$$


Here $W_{\mathbf{k k}^{\prime}}$ is the probability for scattering from the potential $V$ from the state with $\boldsymbol{k}$ to the state with $\boldsymbol{k}^{\prime}$.

One can see from the Eq. (5) that $\rho^{\prime}$ relaxes in the time which is of order of momentum relaxation time, but $\bar{\rho}$ relaxes in the longer time which is determined by $H^{\prime}$ - see (6). At these long times, the Eq. (5) reduces to a quasistationary one:

$$
\sum_{\mathbf{k}^{\prime}} W_{\mathbf{k k}^{\prime}}\left[\rho^{\prime}(\boldsymbol{k})-\rho^{\prime}\left(\boldsymbol{k}^{\prime}\right)\right]=-\frac{i}{\hbar}\left[H^{\prime}, \bar{\rho}\right] .
$$

Finding $\rho^{\prime}(\boldsymbol{k})$ from here and substituting it to the Eq. (6), one can obtain the closed equation for $\bar{\rho}$ :

$$
\frac{\partial \bar{\rho}}{\partial t}=-\frac{1}{\hbar^{2}} \sum_{n} \tau_{n}\left[H_{-n}^{\prime},\left[H_{n}^{\prime}, \bar{\rho}\right]\right]
$$

Here

$$
H_{n}^{\prime}=\oint \frac{d \varphi_{\mathbf{k}}}{2 \pi} H^{\prime}(\boldsymbol{k}) \exp \left(-i n \varphi_{\mathbf{k}}\right)
$$

where $\varphi_{\mathbf{k}}$ is the angle between $\boldsymbol{k}$ and $x$ axis, and

$$
\frac{1}{\tau_{n}}=\oint d \theta W_{\mathbf{k k}^{\prime}}(1-\cos n \theta),
$$

where $\theta=\varphi_{\mathbf{k}}-\varphi_{\mathbf{k}^{\prime}}$. The Eq. (8) clearly demonstrates that it is spin-orbit interaction which causes $\bar{\rho}$ relaxation.

After substituting $\bar{\rho}$ in a form

$$
\bar{\rho}=f_{0}+\frac{1}{2} \boldsymbol{\sigma} \cdot \mathfrak{x}
$$

the Eq. (8) reduces to following equations:

$$
\begin{gathered}
\dot{f}_{0}(\varepsilon, t)=0, \\
\dot{æ}_{i}(\varepsilon, t)=-\Gamma_{i j}(\varepsilon) æ_{j}(\varepsilon, t),
\end{gathered}
$$

where

$$
\Gamma_{i j}=-\frac{1}{\hbar^{2}} \operatorname{Tr}\left\{\sum_{n} \tau_{n}\left[H_{-n}^{\prime},\left[H_{n}^{\prime}, \sigma_{j}\right]\right] \sigma_{i}\right\} .
$$

An initial condition may be derived considering the spin dynamics after the time $\tau_{n}$, but before the spin relaxation time. In the time $\tau_{n}$, the density matrix becomes isotropic but the spin relaxation processes do not start yet. Therefore [8]:

$$
\begin{aligned}
& f_{0}(\varepsilon)=\frac{1}{2}\left[F_{+}(\varepsilon)+F_{-}(\varepsilon)\right], \\
& æ(\varepsilon)=\boldsymbol{s}\left[F_{+}(\varepsilon)-F_{-}(\varepsilon)\right],
\end{aligned}
$$

where $s$ is the unit vector along the spin and $F_{ \pm}(\varepsilon)$ are distribution functions of particles with the spin projection on $s$ equal to $\pm 1 / 2$.
Taking into account that the spin density, $\boldsymbol{S}(t)$, is the average of $æ$ over $\varepsilon$, one can obtain the kinetic equation for it at the time longer than $\tau_{n}$ :

$$
\dot{S}_{i}=-\frac{S_{j}}{\tau_{i j}},
$$

where the tensor of reciprocal spin relaxation times is:

$$
\frac{1}{\tau_{i j}}=\frac{\int d \varepsilon\left[F_{+}(\varepsilon)-F_{-}(\varepsilon)\right] \Gamma_{i j}(\varepsilon)}{\int d \varepsilon\left[F_{+}(\varepsilon)-F_{-}(\varepsilon)\right]} .
$$

The Eq. (17) represents the extension of the results of Ref. [5] to the case of an arbitrary spin-orbit interaction $H^{\prime}$ and takes into account the anisotropy of scattering.

Substituting $H^{\prime}=H_{1}+H_{2}$ into (13) and then $\Gamma_{i j}$ into (17) we have:

$$
\begin{gathered}
\frac{1}{\tau_{z z}}=C\left(a_{1}^{2}+a_{2}^{2}\right), \frac{1}{\tau_{z x}}=\frac{1}{\tau_{z y}}=0, \\
\frac{1}{\tau_{x x}}=\frac{1}{\tau_{y y}}=\frac{C}{2}\left(a_{1}^{2}+a_{2}^{2}\right), \frac{1}{\tau_{x y}}=-C a_{1} a_{2},
\end{gathered}
$$

where

$$
C=\frac{8 m}{\hbar^{4}} \frac{\int d \varepsilon\left[F_{+}(\varepsilon)-F_{-}(\varepsilon)\right] \tau_{1}(\varepsilon) \varepsilon}{\int d \varepsilon\left[F_{+}(\varepsilon)-F_{-}(\varepsilon)\right]} .
$$

Equations 18 - 20) generalize the results 3,4,5 for the case of two spin relaxation mechanisms.

\section{DISCUSSION}

It follows from the Eq. (19) that because of the two mechanisms $\left(a_{1} \cdot a_{2} \neq 0\right)$, the spin relaxation in the plane of the QW becomes anisotropic. It should be emphasized that if there is only one mechanism $\left(a_{1} \cdot a_{2}=0\right)$, then the spin relaxation is isotropic in spite of the cubic symmetry of the Hamiltonian $H_{1}$ or $H_{2}$. Thus the cubic anisotropy of the real QW structure manifests itself due to the interference of two spin relaxation mechanisms.

The system (16) may be rewritten as follows:

$$
\dot{S}_{x} \pm \dot{S}_{y}=-\frac{S_{x} \pm S_{y}}{\tau_{ \pm}},
$$

where

$$
\frac{1}{\tau_{ \pm}}=\frac{C}{2}\left(a_{1} \pm a_{2}\right)^{2} .
$$

The times $\tau_{+}$and $\tau_{-}$describe the relaxation of the spin oriented along the directions [110] and [110] respectively.

The most bright manifestation of spin relaxation anisotropy occurs if $a_{1}= \pm a_{2}$. In this case, one of the times $\tau_{+}$or $\tau_{-}$becomes infinite. Therewith the other is equal to $\tau_{z z}$. 
Efficiency of the mechanisms depends on both the material and the shape of the QW. It was shown that in a rectangular GaAs/AlGaAs QW, the mechanism (11) dominates [9], and in an asymmetrical InGaAs/AlAs QWs the mechanism (2) is the most important [10] or they are comparable [4].

It is clear that if both $a_{1}$ and $a_{2}$ are not equal to zero, then the spin sublevels split. In the work [11] it is shown that $a_{1}$ and $a_{2}$ may be comparable in magnitude and, hence, the spin splitting is strongly anisotropic.

The spin relaxation anisotropy results from the initial $T_{d}$ symmetry of the zinc-blende semiconductor. For this reason, the similar effect can take place in a strained bulk crystal. The corresponding Hamiltonian linear in $3 \mathrm{D}$ wave vector, $\boldsymbol{k}$, and components of an elastic strain tensor, $u_{i j}$, has the form:

$$
\begin{array}{r}
H^{\prime}(u)=A_{1} u_{i i}\left(\sigma_{i+1} k_{i+1}-\sigma_{i+2} k_{i+2}\right) \\
+A_{2} u_{i j}\left(\sigma_{i} k_{j}-\sigma_{j} k_{i}\right) .
\end{array}
$$

Here $i, j=x, y, z, i+3 \rightarrow i, A_{1}$ and $A_{2}$ are constants. Doing calculations for $1 / \tau_{i j}$ in a way similar to (17), one can obtain three different spin relaxation times. It can be shown that the maximum anisotropy may be achieved if

$$
A_{1} u_{x x}=A_{1} u_{y y}=-A_{1} u_{z z} / 2=A_{2} u_{x y} / 3
$$

with the rest of $u_{i j}=0$. Therewith two spin relaxation times are equal to each other and the third is infinite. Note that the tensor $u_{i j}$ determined by (24) may be obtained by applying two uni-axial strains along the axes [001] and [110] and they are not restricted to uni-axial strain along any axes.

\section{CONCLUSION}

The possibility for spin relaxation suppression was noted in Ref. [5] for a QW grown along [110] direction when the spin is oriented along the same axis. The present work shows that the spin relaxation rate also decreases for [110] direction, but in a QW grown in the symmetrical direction [001]. Therefore this decrease takes place for the spin lying in the plane of the QW.

Analysing weak localisation effect, the authors of Refs. [3] showed that the mechanisms (11) and (2) suppress each other in anomalous magnetoresistance, but they are additive in spin relaxation. The present analysis shows that the suppression occurs in the spin relaxation also. Besides, we have found that spin relaxation is anisotropic even in the plane of the QW.

We thank U. Rössler, M.I. Dyakonov and A.N. Titkov for fruitful discussions.

This work was financially supported by Russian Foundation for Basic Research (grant 98-02-18424) and pro- gramm "Physics of Solid State Nanostructures" (grant 97-1035).

[1] Optical orientation, edited by F. Meier, B.P. Zakharchenya (North-Holland, Amsterdam, 1984).

[2] S. Hikami, A. Larkin, and Y. Nagaoka, Progr. Theor. Phys. 63, 707 (1980).

[3] F.G. Pikus and G.E. Pikus, Phys. Rev. B 51, 16928 (1995).

[4] W. Knap, C. Skierbiszewski, A. Zduniak, E. LitvinStaszevska, D. Bertho, F. Kobbi, J.L. Robert, G.E. Pikus, F.G. Pikus, S.V. Iordanskii, V. Moser, K. Zekenes, and Yu.B. Lyanda-Geller, Phys. Rev. B 53, 3912 (1996).

[5] M.I. Dyakonov and V.Yu. Kachorovskii, Fiz. Techn. Poluprov. 20, 178 (1986) [Sov. Phys. Semicond. 20, 110 (1986)].

[6] Yu.L. Bychkov and E.I. Rashba, J. Phys. C 17, 6093 (1984).

[7] G.E. Pikus and A.N. Titkov in [1].

[8] M.I. Dyakonov and V.I. Perel', Fiz. Tverd. Tela 13, 3581 (1971) [Sov. Phys. Solid State 13, 3023 (1972)].

[9] D. Stein, K. von Klitzing, and G. Weimann, Phys. Rev. Lett. 51, 130 (1983).

[10] A.M. Kreshchuk, S.V. Novikov, T.A. Polyanskaya, and I.G. Savel'ev, Semicond. Sci. Technol. 13, 384 (1998).

[11] L. Wissinger, U. Rössler, R. Winkler, B. Jusserand, and D. Richards, Phys. Rev. B 58, 15375 (1998). 